\title{
ESTILOS DE APRENDIZAJE Y SU ADAPTACIÓN A RECURSOS DE LAS TECNOLOGÍAS DE LA INFORMACIÓN Y LA COMUNICACIÓN (TIC) EN LA ENSEÑANZA DE LENGUAS EXTRANJERAS
}

Learning Styles and their Adaptability to Information and Communication Technologies in Foreign Language Teaching

PAZ DíEz ARCón

UNED, España

\begin{tabular}{l} 
KEY WORDS \\
\hline Learning Styles \\
Adaptation \\
ICT \\
Foreign Languages \\
Teaching
\end{tabular}

PALABRAS CLAVE

Estilos de Aprendizaje Adaptación

TIC

Enseñanza de Idiomas Lenguas Extranjeras
ABSTRACT

Virtual learning is offered to students as part of didactic programs where tasks performed through ICT may not consider the variety of existing learning profiles or styles and turn out to be not sufficient for some of the learners. The study presents the use and profile of the different learning styles applied to foreign language teaching and their interaction with didactic materials in regard to the use of ICT resources and their different applications. 


\section{Introducción}

$\mathrm{E}$ l aprendizaje de lenguas extranjeras forma parte de la virtualización en la enseñanza. Los docentes hacen cada vez más uso de las TIC, para que los estudiantes puedan percibir el proceso educativo de manera más amena y dinámica, lo que lleva a una cierta mejoría de los procesos didácticos (Zayas, 2014.). Sin embargo, el aprendizaje virtual, donde las interacciones se dan parcial o totalmente en espacios on-line a través de las TIC (Area y Adell, 2009), se ofrece al estudiante como parte de programas didácticos en los que las tareas llevadas a cabo a través de las TIC, pueden no tener en cuenta los diferentes perfiles o estilos de aprendizaje del alumnado, y por lo tanto, resultar insuficientes para el aprovechamiento óptimo de parte de ellos.

El estudio persigue presentar el uso y perfil de los diferentes estilos de aprendizaje aplicados a la enseñanza de las lenguas extranjera, según la dimensión social, es decir, aquella que tiene en cuenta la preferencia del estudiante en su interacción con los materiales didácticos y su relación con el uso de las TIC en sus diversas aplicaciones. El objetivo principal es localizar herramientas tecnológicas propicias para cada estilo, que puedan tener impacto en el proceso de enseñanza-aprendizaje. Derivado de éste, surgen dos objetivos secundarios: adaptar los recursos de manera personalizada para que el proceso de aprendizaje sea más eficiente y efectivo, y localizar las posibles dificultades en relación a las TIC utilizadas, que se encuentran los estudiantes durante la instrucción para la propuesta de soluciones.

Las herramientas que se van a emplear son una selección de recursos tecnológicos que, en base a su aplicabilidad (Vázquez y Martín, 2014), pueden ser usados en el aprendizaje de una lengua extranjera.

\section{Marco teórico}

En el aprendizaje de idiomas y para que este se desarrolle de manera óptima, es necesario tener en cuenta los estilos de aprendizaje, es decir, las diferentes maneras que tiene una persona de aprender, recogiendo los aspectos pedagógicos y cognitivos a los que se enfrenta (Casero, 2016). Se parte de la base de que cada estudiante tiene ritmos diferentes de aprendizaje (Montes, 2005). Como consecuencia de los diferentes estilos de aprendizaje, se responde de manera diversa a los recursos que se ofrecen como parte del material didáctico, por lo que una buena adecuación de estos, puede ser decisiva para que el estudiante esté más motivado ante el estudio del idioma, y así lograr enriquecer su proceso formativo.

En referencia a los estilos de aprendizaje, investigadores en la materia como Dunn y Dunn (1984), determinan que es necesaria la detección de los estímulos que favorecen el buen aprendizaje de manera individual, y saber dar respuesta a ellos. En total presentan 18 variables que pueden afectar a la forma que tiene el individuo para aprender. Según la teoría de Kolb (1984) el aprendizaje está ligado a la experiencia y desarrolla su teoría en base a esta afirmación, por lo que aspectos socioculturales, experiencia previa $y$ factores hereditarios, afectan directamente al perfil del estudiante. Su modelo se estructura en dos niveles: el ciclo de aprendizaje en cuatro etapas por las que todo estudiante debe pasar: experiencia previa (tener una experiencia); observación reflexiva (sobre esa misma experiencia); conceptualización abstracta (conclusiones y aprendizaje como resultado de la experiencia); y por último, la experimentación activa (probar en la práctica lo aprendido). El segundo nivel que trabaja el autor es determinar en sí, cuatro estilos de aprendizaje teniendo en cuenta los procesos cognitivos internos de cada estudiante. La clasificación y características de cada perfil, detalladas a continuación de manera más amplia, ayudan al docente para la adecuación de materiales en el intento de optimizar los recursos:

- Acomodador: se basan en la intuición, más que en la lógica, por lo que los estudiantes con este perfil tienden a preferir enfoques prácticos y a ser receptivos ante nuevos retos, antes que profundizar en el análisis lógico. Tienen facilidad para adaptarse, y se pueden mostrar impacientes.

- Divergente: poseen capacidad imaginativa, por lo que destacan en la producción de ideas y facilidad para considerar situaciones específicas vistas desde diferentes perspectivas. Recopilan información y usan la imaginación para la resolución de problemas. 
- Convergente: organizan el conocimiento a través del razonamiento hipotético-deductivo, usan su aprendizaje para resolver problemas prácticos y tomar decisiones encontrando soluciones a preguntas y/o problemas.

- Asimilador: desarrollan la capacidad de crear modelos teóricos mediante el razonamiento inductivo, por lo que el interés se enfoca en las ideas y conceptos abstractos, más alejados de la práctica.

Honey y Mumford (1986), plantean el por qué ante un mismo contexto, unos estudiantes aprenden, y otros no. Parten de la teoría de Kolb para dar respuesta a esta cuestión, estableciendo que las reacciones son diferentes, dependiendo de las necesidades de aprendizaje de cada uno. Se formulan cuatro estilos, que tienen estrecha relación con los propuestos por Kolb, siendo los rasgos de cada uno equivalentes a los mencionados: activo (antes: acomodador); reflexivo (antes: divergente); teórico (antes: asimilador); y pragmático (antes: convergente).

Por último, los estudios realizados por Salas y Cabrera (2014), enfocan los estilos de aprendizaje desde una perspectiva personológica. Se asume que el proceso de aprendizaje que marca los diferentes perfiles vienen dados por hábitos, habilidades, y capacidades, que tienen su origen en las particularidades de los procesos psíquicos o niveles de conocimiento integradas con formaciones psicológicas inductoras a diferentes niveles de complejidad en el funcionamiento de la personalidad del que aprende (Aguilera y Ortiz, 2009).

En referencia a los recursos virtuales en el aprendizaje de lenguas extranjeras, estudios realizados sugieren que las plataformas online facilitan de manera natural el aprendizaje de una segunda lengua y sus habilidades y competencias comunicativas (McBride, 2009); y se mantiene la idea de que los estudiantes pueden usar las plataformas virtuales para encontrar herramientas que se ajusten a sus necesidades de aprendizaje (Horwitz, 2020). Por otra parte, investigadores en el aprendizaje de lenguas extranjeras, mantienen que las llamadas "Social Network Sites", tienen potencial considerable para el desarrollo de la pedagogía L2 (McBride, 2009). Por lo que cobra importancia el hecho de facilitar la labor de búsqueda de tareas o dinámicas en una segunda lengua acordes al estilo propicio de aprendizaje del alumno, sin por ello devaluar el propio trabajo autónomo que han de adoptar los nuevos perfiles de estudiante, donde: "estos poseen un conocimiento cercano a las tecnologías, emplean nuevos hábitos de comunicación, se interesan por nuevas formas de enseñanza y autoaprendizaje en línea, demandan sistemización en la transmisión de conocimientos, y se preocupan por ser competitivos" (Romero y Crisol, 2012). El docente puede presentar entonces, teniendo en cuenta estos factores: tareas adaptadas a los perfiles de aprendizaje que funcionen como complemento a la enseñanza de idiomas; o directamente integrarlas en las propias unidades didácticas de las que se componen los cursos. Los blogs y wikis son un ejemplo de recursos que han sido probados como experiencias exitosas de utilización de materiales $\mathrm{y}$ herramientas digitales en lenguas extranjeras. Armstrong y Retterer (2008) afirman que los blogs ayudan a trabajar aspectos gramaticales entre otros aspectos positivos, mientras que Kessler y Bikowski (2010) constatan que las wikis promueven la autonomía en el aprendizaje (Vázquez y Martín, 2014).

La selección de las herramientas TIC que se integran en el proceso de aprendizaje, necesitan ser analizadas y contrastarlas con los objetivos que se tienen marcados para los estudiantes, incluyendo las necesidades como grupo, y enfoque(s) metodológico(s) que se pretenden aplicar en el proceso enseñanza-aprendizaje (Molina, 2016). A esto hay que añadirle el ajuste de estas mismas herramientas a las necesidades individuales del estudiante para poder proponer $\mathrm{y}$ facilitarle recursos TIC personalizados. La organización y selección de las actividades y recursos online, tiene que ser llevada a cabo por docentes competentes que puedan hacer uso de las herramientas de manera efectiva y eficiente (Son, 2011). Con ello se tiene que asegurar que las tareas adaptadas sean coherentes con el resto del material y su contenido.

\section{Método}

Se plantea un estudio correccional aplicando el método inductivo dada la naturaleza de sus objetivos. Se pretende medir la relación que existe entre dos o más variables, en el contexto dado. E 
intentará determinar si hay una correlación, el tipo de correlación y su grado o intensidad (Abreu, 2012). La duración del curso será de 120 horas, por lo que se puede considerar una investigación diacrónica, ya que se pretende verificar los cambios que se puedan producir durante la instrucción y desarrollo de este.

\section{Contexto y sujetos}

El estudio pretende tener lugar entre estudiantes de la Escuela Oficial de Idiomas de la Comunidad de Madrid, enmarcados en el nivel B2 (usuario independiente), donde se le presupone un conjunto de habilidades adquiridas en la segunda lengua. La selección de participantes será una muestra poblacional de 50 personas, donde el número de mujeres y hombres sea equivalente. Sobre esta muestra, se podrán obtener datos personales de los individuos que puedan ser reflejados en los resultados de manera estadística como la edad y el nivel de estudios (estudios superiores, bachiller, formación profesional, etc.). Por otra parte, será interesante determinar de manera individual la(s) motivación(es) para el estudio de la segunda lengua: ampliación de estudios, motivos laborales, aprendizaje por placer, viajar, refrescar los conocimientos, etc. Por cuestiones éticas, se garantiza que los datos con información personal que se solicitan, serán anonimizados. Se solicitará consentimiento explícito sobre la participación de los estudiantes en el estudio, y se les proveerá información completa sobre el diseño y su papel en el estudio. El conjunto de estudiantes será informado de los riesgos y beneficios que conlleva la asignación de las aulas, estableciéndose en una de ellas un grupo de control.

De esta forma, se asegura que comprenden toda la información relativa a este, y se confirma su participación voluntaria y conocimiento sobre la posibilidad de retirarse en cualquier momento.

\section{Instrumento}

Para definir los estilos de aprendizaje de cada estudiante, se utilizará el Cuestionario HoneyAlonso de Estilos de Aprendizaje CHAEA (2007) (Anexo 1), el cual cuenta con 80 ítems, que determinan el perfil de aprendizaje de la muestra poblacional. Siguiendo la clasificación de los autores, basada en la investigación de Kolb (1984), los resultados se verán reflejados en número de estudiantes enmarcados en cada perfil, lo que definirá la configuración de las aulas (A y B). Al conjunto de estudiantes se les asignará un nombre identificativo para asegurar la protección de datos personales en la presentación de resultados. Esta asignación se realizará de acuerdo al número de estudiantes resultante de cada perfil, teniendo que tener cada uno de estos rasgos identificativos. Por ejemplo, para el estudiante activo del aula A: AA1, AA2...etc., o para el estudiante reflexivo de la clase B: RB1, RB2 ...etc. Durante el curso, los estudiantes trabajarán un total de 20 unidades didácticas, siendo ejemplo de una de ellas la reflejada en el Anexo 2 (última unidad didáctica del curso). Dentro del método cuasiexperimental aplicado en el estudio, se aplicará el posttest, donde se presenta como instrumento, la evaluación sumativa de la unidad didáctica final incluida en la sesión 3 de la unidad (ver Anexo 2). Esta, estará adaptada al nivel establecido y se aplicará al conjunto de estudiantes que forman parte de la investigación. El desarrollo de las sesiones del curso y posteriores evaluaciones, se realizarán en dos aulas que engloban el conjunto de la muestra poblacional, y que van a trabajar las mismas unidades didácticas. La primera (A), estará compuesta por la mitad de estudiantes enmarcados en cada perfil, los cuales serán seleccionados para ser parte de este aula por sorteo, y utilizarán los recursos TIC adaptados a sus perfiles determinados previamente, como material adicional durante el desarrollo de las sesiones 1 y 2 que componen cada unidad didáctica (ejemplos en el Anexo 2). El segundo grupo (B) constará de la otra mitad de alumnos. Podrán utilizar los mismos recursos, pero de manera aleatoria (no adaptados), y desde su propia iniciativa. Mediante la evaluación sumativa final, se verificará en qué grado la instrucción ha sido alcanzada, y que aula consigue mejores resultados en el cómputo final.

Se hará uso de un total de 4 herramientas online para la enseñanza de idiomas adaptadas a los diferentes perfiles. Para determinar la validez de los criterios seguidos para la correcta asociación de los diferentes perfiles con su 
herramienta correspondiente, se realizará una consulta a un grupo de expertos conformado por especialistas en psicología, educación y filología. Los resultados de la consulta no deberán ser inferiores al $90 \%$ en la confirmación del uso favorable de los recursos mencionados ligados a cada perfil. Con esta consulta se pretende la reafirmación de la validez y la fiabilidad (Robles y Rojas, 2015).

La selección de los recursos TIC está determinada por las características definitorias de los diferentes perfiles detallados previamente, y su relación con las propias particularidades de los recursos. Por lo que se han tenido en cuenta los factores que favorecen y desfavorecen el aprendizaje en cada estilo, y se han adaptado a las posibilidades que ofrecen los recursos mencionados.

Rationale: relación entre estilos de aprendizaje y recursos TIC escogidos para el estudio

- Activo-Uso de redes sociales (Facebook/Instagram) Los estudiantes enmarcados en el estilo activo se preguntan el cómo. Aprenden con más facilidad cuando se les presenta un reto o desafío, y cuando realizan tareas en los que el resultado es inmediato, lo pueden compartir sin limitaciones formales, y pueden participar activamente. No les favorece el trabajar solos, ni analizar e interpretar datos. (Honey y Alonso, 1999). Mediante el uso de las redes sociales mencionadas, por una parte el alumno puede compartir dudas y experiencias sobre las sesiones, compartir material adicional, etc.; y el docente puede elaborar cuestiones sobre el tema que se está tratando en clase, que requieran respuestas cortas y precisas, y fomenten el debate poco profundo que conlleva llevarlo a cabo en estas plataformas, estimulando así las fortalezas del estudiante "activo".

- Reflexivo - Blog. Los estudiantes reflexivos se preguntan el porqué. Optimizan el aprendizaje cuando pueden adoptar la postura de observador, y se les da posibilidad de pensar antes de actuar, no trabajan bien ante la no planificación y la premura (Honey y Alonso, 1999). El blog educativo, y más específicamente el de alumno (son solo ellos los que pueden realizar entradas y compartir experiencias), es el material que se adecua el estilo descrito, ya que no solo brinda apoyo al estudiante y al educador en el proceso de aprendizaje, facilitando la reflexión, el cuestionamiento de sí mismo, y de los otros; sino que proporciona contextos que fomentan el análisis y la crítica. (Vázquez y Martín, 2014).

- Teórico - Wiki. Los teóricos indagan sobre el qué. Valoran la lógica y la racionalidad, por lo que se sienten más cómodos ante modelos, teorías, y conceptos que supongan desafío, sin embargo, no rinden ante falta de fundamento teórico o ambigüedad. (Honey y Alonso, 2014). Mediante el uso de las wikis, pueden recopilar información, ordenar materiales, elaborar glosarios, etc. (Vázquez y Martín, 2014). Dinámicas que promueven las fortalezas del estudiante "teórico", ya que la organización obtenida con su uso, da pie al desarrollo del análisis y la síntesis estructurada, y de la lógica basada en datos.

- Pragmático - WebQuest. Los perfiles pragmáticos se cuestionan el qué pasaría si...Trabajan bien con tareas que relacionen teoría y práctica, y cuando pueden probar de inmediato lo que han aprendido. Les cuesta continuar tareas que no tienen finalidad aparente (Honey y Alonso, 1999). Las WebQuest son una dinámica que compatibiliza con el modo de aprendizaje de este perfil, ya que consisten en el planteamiento de un problema, que el alumno, usando recursos interactivos preestablecidos, tiene que resolver. Fomenta la investigación del material presentado, y desarrolla habilidades transversales. (Vázquez y Martín, 2014).

Tabla 1: Resumen asociación de estilos de aprendizaje y recursos TIC.

\begin{tabular}{|c|c|}
\hline ESTILO DE APRENDIZAJE & HERRAMIENTA TIC \\
\hline Activo & $\begin{array}{c}\text { Redes sociales } \\
\text { (Facebook/Instagram) }\end{array}$ \\
\hline Reflexivo & Blog \\
\hline Teórico & Wiki \\
\hline Pragmático & WebQuest (adaptada) \\
\hline
\end{tabular}

Fuente: Elaboración propia. 


\section{Resultados}

Los resultados se presentarán por medio de tablas que han de representar la calificación media obtenida por cada perfil en las aulas A y B en el posttest o prueba final, $y$ su correspondiente explicación más exhaustiva. Con esta información se podrá deducir si el uso de recursos TIC adaptados a los estilos de aprendizaje tiene efecto positivo en los estudiantes que lo han experimentado. Se espera que si es así, los resultados obtenidos del resto de evaluaciones durante el transcurso de las sesiones, sean más significativos de manera progresiva al estar el uso de las herramientas más consolidado a medida que avanza la instrucción. Así quedará reflejado especificando los resultados independientes de las evaluaciones incluidas en cada unidad didáctica. Habrá que tener en cuenta la posibilidad de que algún estudiante del aula $B$, decida utilizar herramientas de refuerzo que casen con su perfil de manera involuntaria, para ello habrá que recoger información sobre qué estudiantes de ese aula han usado recursos adicionales, y cuáles; e incluir esta información para el análisis de los datos y contabilizarlos como perfil adaptado. Posteriormente, se añadirán en base a los resultados individuales de estudiantes A y B, datos estadísticos descriptivos para presentar información adicional relevante.
Por otra parte, para la verificación de correlaciones se usará el chi-cuadrado para contrastar la hipótesis de independencia entre las variables, dada la naturaleza no continua de todas las variables al ser el estilo de aprendizaje y las herramientas TIC variables categóricas.

\section{Conclusiones}

Las conclusiones se presentarán en base a los resultados que han de determinar la validez de los objetivos del estudio tras su implementación: localizar y adaptar herramientas tecnológicas adecuadas a los estilos de aprendizaje y lograr que mediante la adaptación mejore el proceso de aprendizaje. También localizar dificultades en relación al uso de las TIC durante el periodo en el que transcurre la formación en la segunda lengua, para la propuesta de soluciones.

Si los datos demuestran que la hipótesis es válida, se remarcará que es necesario replicar el estudio con múltiples variables de cada uno de los elementos que componen el estudio, ya que los datos obtenidos solo permitirían intuir una tendencia que tiene que ser corroborada de manera más amplia. Esta propuesta de replicabilidad podrá abrir las puertas a futuras investigaciones, enfocándose en otro tipo de recursos TIC adaptados y con la posibilidad de emplearlos durante diferentes periodos de tiempo, o seleccionar participantes con niveles del idioma y edades diferentes. 
Estilos de Aprendizaje y su adaptación a recursos de las Tecnologías de la Información y la Comunicación (TIC)...

\section{Referencias}

Abreu, J. (2012). Hipótesis, método \& diseño de investigación (hypothesis, method \& research design). Daena: International Journal of Good Conscience, 7(2), 187-197.

Aguilera, E. y Ortiz, E. (2009). Las investigaciones sobre los estilos de aprendizaje y sus modelos explicativos. Revista de estilos de aprendizaje, 2(4).

Alonso, C. M., Gallego, D. y Honey, P. (1999). Los Estilos de Aprendizaje. Bilbao: Ediciones Mensajero. Universidad Deusto.

Area, M. y Adell, J. (2009). E-learning: enseñar y aprender en espacios virtuales. En J. De Pablos (Coord), Tecnología Educativa. La formación del profesorado en la era de Internet. Aljibe, Málaga.

Casero, E.M. (2016). La importancia de las TIC para la enseñanza de idiomas en al aula intercultural (Tesis doctoral no publicada). Departamento de Filologías Extranjeras y sus Lingüísticas, UNED, Madrid.

Dunn, R. (1984). Learning style: State of the science. Theory into practice, 23(1), 10-19.

Franco, P.; Pino Juste, M.R.; y Rodríguez, B. (2009) Tipología y frecuencia del uso de estrategias en el aprendizaje del inglés como lengua extranjera. Enseñanza \& Teaching, 27, 2, 171-191.

Honey, P. y Mumford, A. (1986). The Manual of Learning Styles. Berkshire: Ardingly: House.

Kartal, E. (2005). The Internet and autonomous language learning: a typology of suggested aids. Turkish Online Journal of Distance Education, 4(4), 54-58.

Kolb, D. A. (1984). Experiential learning: Experience as the source of learning and development (Vol. 1). Englewood Cliffs, NJ: Prentice-Hall.

Liu M. et al. (2013) A Study of the Use of Social Network Sites for Language Learning by University ESL Students. In: Lamy MN., Zourou K. (eds), Social Networking for Language Education. New Language Learning and Teaching Environments. Palgrave Macmillan, London

Mackey \& M. Gass, 2005. Second Language Research. Methodology and design. Mahwah, New Jersey: Lawrence Erlbaum Associates.

McBride, K (2009). Social net-working sites in foreign language classes. Opportunities for re-creation. The next generation: Social networking and online collaboration in foreign language learning, 8, 35-38.

McLeod, S.A. (2017, Oct 24). Kolb - learning styles. Simply Psychology.

Molina, I (2016). Algunas herramientas digitales y su uso colaborativo en el aula de ELE. (2020).

Montes, J.M. (2005). Pautas y estrategias para entender y atender la diversidad en el aula. Pulso 28, 199-214.

Moreno, E. y Risueño, J. (2018). Design of a Checklist for Evaluating Language Learning Websites. Porta Linguarum, 30, 23-41.

Robles Garrote, P. y Rojas, M. D. C. (2015). La validación por juicio de expertos: dos investigaciones cualitativas en Lingüística aplicada. Revista Nebrija de Lingüística Aplicada 18.

Romero, M.A. y Crisol, E. (2012). Self-learning guides to teaching as a tool to support teaching.

Salas-Cabrera, J. (2014). Estilos de aprendizaje en estudiantes de la Escuela de Ciencias del Movimiento Humano y Calidad de Vida, Universidad Nacional, Costa Rica. Revista electrónica EDUCARE, 18(3), 159-171.

Son, J. B. (2011). Online tools for language teaching. TESL-EJ, 15(1), 1-12.

Vázquez, E. y Martín, E. (2014). Nuevas tendencias en la elaboración y utilización de materiales digitales para la enseñanza de lenguas. Madrid: McGrawhill Education.

Zayas, F. (2014). Problemas metodológicos en la enseñanza de lenguas extranjeras: ¿son las TIC una solución? Rastros Rostros, 16(30).

\section{Anexos}

\section{Anexo 1}

https://es.scribd.com/document/444017098/Cuestionario-CHAEA-Paz-Diez-Arcon

Anexo 2

https://es.scribd.com/document/443155599/Unidad-Didactica-B2-Paz-Diez-Arcon 